\title{
Family with Sequence Similarity 83 Member H Promotes the Viability and Metastasis of Cervical Cancer Cells and Indicates a Poor Prognosis
}

\author{
Chao Chen ${ }^{1}$, Hua-Feng $\mathrm{Li}^{1}$, Yu-Jie $\mathrm{Hu}^{1}$, Meng-Jie Jiang', Qing-Sheng $\mathrm{Liu}^{2}$, and Jia Zhou ${ }^{2}$ \\ ${ }^{1}$ Department of Radiotherapy, Zhejiang Provincial Hospital of Traditional Chinese Medicine, Hangzhou, Zhejiang; \\ ${ }^{2}$ Department of Geratology, Hangzhou Hospital of Traditional Chinese Medicine, Hangzhou, Zhejiang, China.
}

\begin{abstract}
Purpose: Family with sequence similarity 83 member H (FAM83H) plays key roles in tumorigenesis. However, the specific roles of FAM83H in cervical cancer (CC) have not been well studied.

Materials and Methods: The RNA-seq data of 306 CC tissues and three normal samples downloaded from The Cancer Genome Atlas were used to analyze the expression of FAM83H. The Kaplan-Meier method was used to draw survival curves. Associations between FAM83H expression and clinicopathological factors were analyzed by chi-square test. Cox proportional hazards model was used to analyze prognostic factors. Loss-of-function assays were conducted to discover the biological functions of FAM83H in cell proliferation, colony formation, invasion, and migration. Real-time Quantitative Reverse Transcription PCR (qRT-PCR) and Western blotting were used to measure the expression levels of FAM83H in CC cell lines.

Results: Our results demonstrated that FAM83H is overexpressed in CC tissues and that high FAM83H expression is associated with worse overall survival (OS). High FAM83H expression in CC was associated with clinical stage, pathologic tumor, and pathologic node. Univariate analysis suggested that FAM83H expression was significantly related to the OS of CC patients. Although multivariate analysis showed that FAM83H expression was not an independent prognostic factor for the OS of CC patients, the effects of FAM83H on CC cell growth and motility was significant. Loss-of-function experiments demonstrated that knockdown of FAM83H inhibited proliferation, colony formation, migration, and invasion of CC cells by inactivating PI3K/AKT pathway. Conclusion: FAM83H might play a crucial role in CC progression and could act as a novel therapeutic target in CC.
\end{abstract}

Key Words: Cervical cancer, FAM83H, prognosis, proliferation, migration

\section{INTRODUCTION}

Cervical cancer (CC), the second most common malignancy in females worldwide, is a primary cause of cancer death, leading to about 300000 deaths each year. ${ }^{1}$ Statistically, about 500000 new cases arise per year, and $85 \%$ of the pathologic

Received: December 26, 2018 Revised: April 22, 2019

Accepted: May 9, 2019

Corresponding author: Jia Zhou, MD, Department of Geratology, Hangzhou Hospital of Traditional Chinese Medicine, No. 453 Tiyuchang Road, Hangzhou, Zhejiang 310007, China.

Tel: 86-0571-85827722, Fax: 86-0571-85119481, E-mail: zhoujia_1122@163.com

-The authors have no potential conflicts of interest to disclose

(C) Copyright: Yonsei University College of Medicine 2019

This is an Open Access article distributed under the terms of the Creative Commons Attribution Non-Commercial License (https://creativecommons.org/licenses/ by-nc/4.0) which permits unrestricted non-commercial use, distribution, and reproduction in any medium, provided the original work is properly cited. types are squamous cell carcinoma. In China, CC accounts for $5 \%$ of cancer occurrences and $3.8 \%$ of mortalities in women. ${ }^{2}$ In recent decades, although the cervical cytology screening has facilitated early detection and early treatment, morbidity rates remain high. Worriedly, more and more cases in younger individuals are being recorded. Moreover, the etiology of this cancer is still largely unclear. Thus, many researchers have sought to identify the molecular mechanisms involved in the progression of CC and to seek out more effective tumor therapy strategies.

Family with sequence similarity 83 (FAM83) family members have been shown to possess oncogenic properties, and the expression thereof has been found to be elevated in several human cancer types, including breast cancer. ${ }^{3-5}$ Typically, FAM83 family members are related to poor cancer prognosis. ${ }^{6}$ FAM83 member H (FAM83H) is one member of FAM83. Re- 
cently, it has been reported that FAM83H controls the organization of the keratin cytoskeleton, as well as the formation of desmosomes. ${ }^{7,8}$ In recent research, up-regulation of FAM83H in cancer tissues, compared to normal samples, has been described. For example, FAM83H has been suggested to contribute to the development of colorectal cancer by regulating the organization of the keratin cytoskeleton. ${ }^{8,9}$ Another study also showed that FAM83H might be an important biomarker that can cause androgen independent prostate cancer progression. ${ }^{10}$ Moreover, Kim, et al. ${ }^{11}$ have proposed that FAM83H plays important roles in the progression of hepatocellular carcinoma and is a poor prognostic predictor in hepatocellular carcinoma patients. Nevertheless, the exact role of FAM83H in CC has not been studied to date, and the detailed action mechanisms of FAM83H in CC still remain unknown.

In our study, we conducted in vitro assays to investigate the expression and the mechanisms of FAM83H in the progression of CC. Our results demonstrated that FAM83H is involved in CC cell proliferation and migration, suggesting that FAM83H as a potential novel therapeutic target for CC.

\section{MATERIALS AND METHODS}

\section{Bioinformatic analysis}

The RNA-seq data and clinical data of 306 CC patients and three normal control samples were downloaded from The Cancer Genome Atlas (TCGA, https://tcga-data.nci.Nih.gov/ tcga). The RNA-seq data were used to analyze the expression of FAM83H in CC patients and normal samples. A total of 304 CC patients with detailed clinical information were divided into low or high expression groups according to the median expression of FAM83H. Detailed information on these 304 CC patients is shown in Supplementary Table 1 (only online). The association between the expression of FAM83H and overall survival (OS) was assessed using Kaplan-Meier analysis with log rank test used for comparison. Chi-square $\left(\chi^{2}\right)$ test was used to analyze the association between FAM83H expression and the clinicopathological features of patients with CC. Univariate and multivariate Cox proportional hazards models were performed to evaluate the prognostic value of FAM83H. In addition, we also analyzed the association between expression of FAM83H and OS of CC patients by searching the Human Protein Atlas (https://www.proteinatlas.org) database and Gene Expression Profiling Interactive Analysis (GEPIA, http://gepia.cancer-pku.cn/) database. Patients in the Human Protein Atlas were divided into high or low expression groups with the best cut-off point, while patients in the GEPIA database were divided into high or low expression groups based on the median of FAM83H expression. The data download process complied with all laws and regulations.

\section{Cell culture and transfection}

Human CC cell lines HeLa and SiHa were obtained from the Chinese Academy of Sciences (Shanghai, China). Hacat cells were used as a control. These cells were routinely cultured in RPMI-1640 medium (Gibco, Grand Island, NY, USA) supplemented with $10 \%$ heat-inactivated fetal bovine serum (FBS, Sijiqing Biotec, Hangzhou, China), $100 \mathrm{U} / \mathrm{mL}$ penicillin, and $0.1 \mathrm{mg} / \mathrm{mL}$ streptomycin (Sigma Chemical Company, Shanghai, China) at $37^{\circ} \mathrm{C}$ with $5 \% \mathrm{CO}_{2}$ in a humidified incubator. Nonadherent cells were washed away after 3 days, and adherent cells were incubated with fresh complete medium. The cells were sub-cultured at the split ratio of 1:2 after reaching confluence.

Two FAM83H-specific small interfering RNAs (siFAM83H\# 1: 5' -GAGGAAGUCCAACUACAGG-3' and siFAM83H\#2: 5' - CUACAGGAUUUAUCGAGCA-3') were synthesized by GenePharma (Shanghai, China). Lipofectamine 2000 transfection reagent (Invitrogen, Carlsbad, CA, USA) was used for transfection. Twenty-four hours after transfection, the HeLa cells were lysed and then subjected to Real-time Quantitative Reverse Transcription PCR (qRT-PCR) to examine the efficiency of inhibition. HeLa cells transfected with scrambled siRNA control (si-con) were regarded as controls.

\section{RNA extraction and qRT-PCR}

Total RNA was extracted using TRIzol Reagent (Invitrogen, Carlsbad, CA, USA) from CC cells transfected with siRNA and the negative controls. The extracted RNA was dissolved using RNAase-free water that was treated with diethyl pyrocarbonate. Next, cDNA was synthesized based on total RNA using PrimeScript RT reagent Kit (TaKaRa, Dalian, China). Afterwards, qRT-PCR was conducted to quantify the expression levels of FAM83H. The primers for this gene were as follows: FAM83H, F: 5' - CTGGACCAGAAGATGCCCCT-3', and R: 5'CTGGAGGAGATGGCAGACCT-3'. Glyceraldehyde-3-phosphate dehydrogenase (GAPDH) was used as an endogenous control (F: 5'-GGAGCGAGATCCCTCCAAAAT-3', R: 5'-GGCT GTTGTCATACTTCTCATGG-3'). The $2^{-\Delta \Delta C T}$ method was utilized to calculate the relative expression levels.

\section{Cell proliferation and colony formation assays}

Cell proliferation ability was assessed using a Cell-Counting Kit 8 (CCK-8) assay, as described by the manufacturer. The HeLa cells were cultured in 96-well plates, and then, $10 \mu \mathrm{L}$ of CCK-8 solution was added to each well at the indicated time points $(24,48,72$, and $96 \mathrm{~h})$ after transfection. Further, cells were cultured for $2 \mathrm{~h}$ at $37^{\circ} \mathrm{C}$ in a $5 \% \mathrm{CO}_{2}$ incubator. Absorbance was measured at $450 \mathrm{~nm}$.

Colony formation was conducted as described previously. ${ }^{12,13}$ Specifically, si-FAM83H and si-control cells were cultured in medium supplemented with $10 \%$ FBS, and replacing the medium every four days. Then, the cells were incubated at $37^{\circ} \mathrm{C}$ in a $5 \% \mathrm{CO}_{2}$ incubator for 1 week. Subsequently, $4 \%$ paraformaldehyde was used to fix the colonies, and $0.1 \%$ crys- 
tal violet (Sigma, St. Louis, MO, USA) was applied to stain the colonies. The images of the colonies were photographed. The number of colonies were quantified using Image J software (National Institutes of Health, Bethesda, MD, USA). The number of colonies in the si-con group was defined as $100 \%$.

In vitro woundhealing, migration, and invasion assays The effect of FAM83H on the migration of HeLa cells was measured using wound-healing assay kits. Wound healing inserts were added into 24-well plates, and the cells were seeded to either side of the insert after $24 \mathrm{~h}$ transfection and cultured overnight to form a monolayer. After that, inserts were discarded when an open wound field in the monolayer of cells was generated. Then, cells continued to be cultured for $24 \mathrm{~h}$ under standard conditions. Photographs of wound healing were recorded with a phase-contrast microscope at 0 and $24 \mathrm{~h}$ after eliminating the inserts.

The migration assay was carried out using a 24-transwell migration chamber with $8-\mu \mathrm{m}$ pore filters (BD Biosciences, San Jose, CA, USA). HeLa cells were added into the migration chamber where the bottom chamber was filled with $500 \mu \mathrm{L}$ of complete medium supplemented with $10 \%$ FBS. For the invasion assay, $2 \times 10^{5}$ cells were seeded in the upper chamber containing an 8- $\mu \mathrm{m}$ pore Matrigel Invasion Chamber (BD Biosciences, San Jose, CA, USA). Serum-free medium was put into the lower chamber in the invasion assay. The migration and invasion chambers were incubated at $37^{\circ} \mathrm{C}$ overnight. Thereafter, the migrated or invaded cells on lower surface of the filter were stained with $0.1 \%$ crystal violet for $20 \mathrm{~min}$ and counted in five microscopic fields for each well.

\section{Western blotting analysis}

Cells were lysed using RIPA buffer (Beyotime, Jiangsu, China), and protein concentration was measured with Bio-Rad DC protein assay (Bio-Rad, Hercules, CA, USA). Then, a total of $20 \mu \mathrm{g}$ of protein from the cell lysate was subjected to sodium dodecylsulphate polyacrylamide gel electrophoresis (SDSPAGE) and transferred to polyvinylidene difluoride membranes. Subsequently, membranes were blocked with 5\% non-fat milk overnight and incubated with the specific primary antibodies (anti-FAM83H, anti-AKT, anti-p-AKT, anti-PI3K, anti-p-PI3K, and anti-GAPDH). Following washing for $30 \mathrm{~min}$ with TBST, secondary antibody was used to incubate the membranes in TBST for $2 \mathrm{~h}$ at room temperature. After washing, the interested protein signals were captured using Enhanced Chemiluminescence Plus detection kit (Thermo Fisher Scientific, Rockford, IL, USA).

\section{Statistical analysis}

One-way analysis of variance (ANOVA) was utilized to compare the values of multi-groups. All experiments were carried out in triplicate. Statistical analysis was performed using SPSS 22.0 software (IBM Corp., Armonk, NY, USA). $p$ values $<0.05$ were considered statistically significant.

\section{RESULTS}

\section{FAM83H elevated in CC}

By retrieving the data in the TCGA database and GEPIA database, we characterized FAM83H expression in CC (Fig. 1A-C). Results revealed marked up-regulation of FAM83H expression in CC tissues, compared to normal tissues (Fig. 1A-C).

In order to verify the bioinformatics results, we next measured the expression of FAM83H in the CC cell lines SiHa and HeLa using qRT-PCR. Consistent with the bioinformatics results, FAM83H expression was remarkably up-regulated in SiHa and HeLa cell lines when compared with normal Hacat cells $(p<0.001)$ (Fig. 1D). We found that expression levels in HeLa cells were significantly higher than those in SiHa cells. Thus, in our study, we selected HeLa cells for subsequent analysis.

FAM83H correlated with clinical characteristics of CC We then analyzed the relationship between FAM83H and clinical pathological characteristics of CC based on the data obtained from TCGA database. The clinicopathological characteristics of CC patients are shown in Table 1. We found that high FAM83H expression in CC was associated with clinical stage ( $p=0.008)$, pathologic tumor $(p=0.023)$, and pathologic node $(p=0.038)$.

FAM83H overexpression related to poor prognosis in CC To further investigate the prognostic value of FAM83H, we performed Kaplan-Meier analysis based on the TCGA, GEPIA, and the Human Protein Atlas databases. The results showed that high FAM83H expression is associated with worse OS in patients with CC based on the TCGA data with the median of FAM83H expression used as the cutoff (Fig. 1E, $p=0.027$ ). Although the difference was not significant, we also noted that high FAM83H expression was associated with lower survival rate to some extent in the GEPIA database (Fig. 1F, $p=0.053)$. From the Human Protein Atlas database, we found that higher expression of FAM83H was associated with shorter survival with the best cut-off point (Fig. 1G, $p=0.004$ ).

Next, we performed univariate and multivariate proportional hazards analyses to identify independent prognostic factors for survival in CC patients. Univariate analysis suggested that FAM83H expression ( $p=0.028)$, clinical stage $(p=0.001)$, pathologic tumor $(p<0.001)$, pathologic metastasis $(p=0.020)$, and pathologic node ( $p=0.003)$ were significantly related to the OS of CC patients (Table 2). Multivariate analysis demonstrated that pathologic tumor was an independent prognostic factor for the OS of CC patients (Table 2) $(p=0.033)$. However, FAM83H expression could not be regarded as independent prognostic factor for the OS of CC patients $(p=0.716)$. Above 

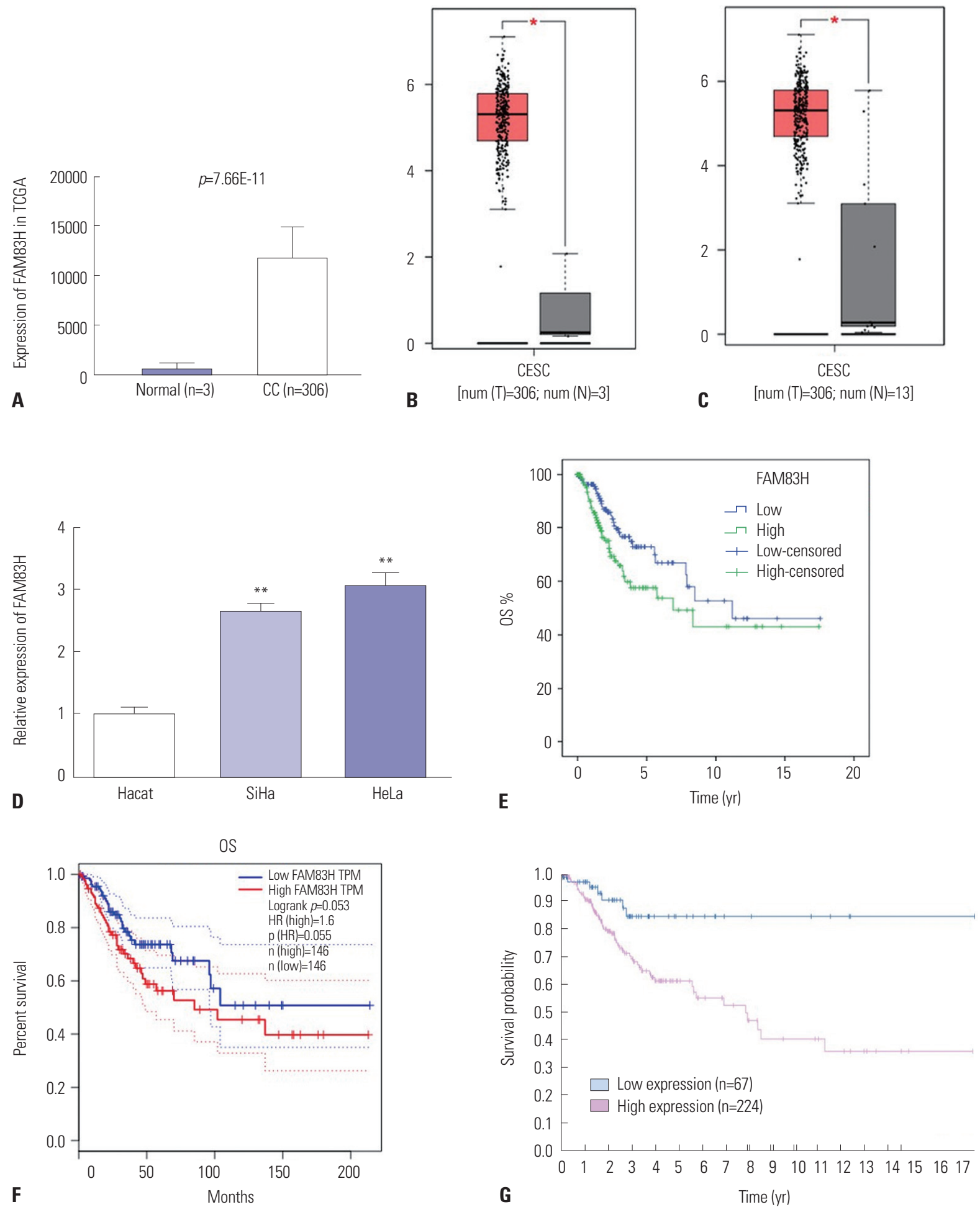

Fig. 1. FAM83H over-expression is associated with poor prognosis of CC. (A) FAM83H expression at mRNA level in CC tissues in TCGA database. (B) FAM83H expression at mRNA level in CC tissues in GEPIA database (match TCGA normal data). (C) FAM83H expression at mRNA level in CC tissues in GEPIA database (match TCGA normal and GTEx data). (D) Real-time Quantitative Reverse Transcription PCR used to measure the expression level of FAM83H in human CC cell lines ( $\mathrm{SiHa}$ and HeLa). The expression of FAM83H was significantly higher in CC cells relative to control cells. (E) KaplanMeier curves of OS of CC patients in TCGA database. Patients were sub-grouped into high $(n=152)$ or low $(n=152)$ groups based on the median of FAM83H expression. $p=0.027$. (F) Kaplan-Meier curves of OS of CC patients in GEPIA database. Patients were sub-grouped into high ( $\mathrm{n}=146$ ) or low $(n=146)$ groups based on the median of FAM83H expression. (G) Kaplan-Meier curves of OS of CC patients in the Human Protein Atlas database. Patients were sub-grouped into high $(\mathrm{n}=224)$ or low $(\mathrm{n}=67)$ groups based on best cut-off point $(p=0.004)$. ${ }^{*} p<0.05,{ }^{* *} p<0.01$. FAM83H, family with sequence similarity 83 member H; CC, cervical cancer; TCGA, The Cancer Genome Atlas; GEPIA, Gene Expression Profiling Interactive Analysis; 0S, overall survival; CESC, cervical squamous cell carcinoma and endocervical adenocarcinoma; TPM, transcripts per million; HR, hazard ratio. 
Table 1. Relationships between FAM83H Expression and Clinicopathologic Features in Cervical Cancer Patients

\begin{tabular}{|c|c|c|c|}
\hline \multirow{2}{*}{ Characteristics } & \multicolumn{2}{|c|}{ Expression of FAM83H } & \multirow{2}{*}{$p$ value } \\
\hline & Low & High & \\
\hline Age (yr) & & & 0.675 \\
\hline$<60$ & 118 & 121 & \\
\hline$\geq 60$ & 34 & 31 & \\
\hline Grade & & & 0.521 \\
\hline $\mathrm{G} 1+\mathrm{G} 2$ & 75 & 78 & \\
\hline G3 & 63 & 56 & \\
\hline Clinical-stage & & & $0.008^{*}$ \\
\hline$|+| \mid$ & 127 & 104 & \\
\hline III+IV & 24 & 42 & \\
\hline Pathologic-T & & & $0.023^{*}$ \\
\hline $\mathrm{T} 1+\mathrm{T} 2$ & 117 & 94 & \\
\hline $\mathrm{T} 3+\mathrm{T} 4$ & 10 & 20 & \\
\hline Pathologic-N & & & $0.038^{*}$ \\
\hline NO & 79 & 54 & \\
\hline N1 & 26 & 34 & \\
\hline Pathologic-M & & & 0.514 \\
\hline MO & 65 & 51 & \\
\hline M1 & 7 & 3 & \\
\hline
\end{tabular}

FAM83H, family with sequence similarity 83 member $\mathrm{H}$.

Since the clinical pathological information was not complete in some cases, the number of cases in grade, Clinical-stage, Pathologic-T, $\mathrm{N}$ and $\mathrm{M}$ are less than 304 , and the number of cases are different. ${ }^{*} p<0.05$. all, the results indicated that, while the expression of FAM83H is significantly associated with OS in CC patients, it cannot be considered an independent prognostic factor.

\section{Knockdown of FAM83H inhibits the proliferation and growth of HeLa cells}

To identify the roles of FAM83H in CC cells, si-FAM83H was transfected into HeLa cells to determine its effect on the proliferation ability of HeLa cells. In the current study, the transfection efficiency of both si-FAM83H \#1 and si-FAM83H \#2 was $>\mathbf{8 0 \%}$ (Fig. 2). In addition, a greater decrease in FAM83H expression was observed in the si-FAM83H \#1 group, compared with the si-FAM83H \#2. Thus, si-FAM83H \#1 was used in subsequent experiments.

CCK-8 results suggested that the knockdown of FAM83H effectively inhibited the proliferation of HeLa cells (Fig. 3) at $48 \mathrm{~h}$ $(p<0.05), 72 \mathrm{~h}(p<0.001)$, and $96 \mathrm{~h}(p<0.001)$. Likewise, colony formation assay showed that FAM83H silencing reduced the colony numbers of HeLa cells ( $p<0.001)$ (Fig. 4).

\section{Knockdown of FAM83H inhibits the migration and invasion of HeLa cells}

Following $24 \mathrm{~h}$ scratch formation in the cell monolayer, the wound in the FAM83H knockdown cells was wider, compared with that in the control cells, suggesting that knockdown of FAM83H significantly inhibits HeLa cell migration (Fig. 5A). Subsequently, we also utilized transwell assay to investigate

Table 2. Univariate and Multivariate Analyses of Clinical Prognostic Factors of Cervical Cancer

\begin{tabular}{|c|c|c|c|c|c|c|}
\hline \multirow{2}{*}{ Variables } & \multicolumn{3}{|c|}{ Univariate analysis } & \multicolumn{3}{|c|}{ Multivariate analysis } \\
\hline & $p$ value & HR & $95 \% \mathrm{Cl}$ & $p$ value & HR & $95 \% \mathrm{Cl}$ \\
\hline FAM83H (low/high) & 0.028 & 1.691 & $1.057-2.706$ & 0.716 & 1.217 & $0.423-3.502$ \\
\hline Clinical-stage (I+II/III+IV) & 0.001 & 2.286 & $1.397-3.743$ & 0.209 & 0.354 & $0.070-1.789$ \\
\hline Pathologic-T (T1+T2/T3+T4) & $<0.001$ & 3.613 & $1.907-6.846$ & 0.033 & 5.214 & $1.141-23.828$ \\
\hline Pathologic-M (M0/M1) & 0.020 & 3.671 & $1.229-10.962$ & 0.988 & 0 & 0 \\
\hline Pathologic-N (N0/N1+N2+N3) & 0.003 & 2.807 & $1.408-5.593$ & 0.137 & 2.164 & $0.783-5.984$ \\
\hline Age $(<60 / \geq 60)$ & 0.054 & 1.641 & $0.991-2.715$ & - & - & - \\
\hline Grade $(\mathrm{G} 1+\mathrm{G} 2 / \mathrm{G} 3+\mathrm{G} 4)$ & 0.681 & 0.896 & $0.530-1.513$ & - & - & - \\
\hline
\end{tabular}

$\mathrm{HR}$, hazard ratio; $\mathrm{Cl}$, confidence interval; FAM83H, family with sequence similarity 83 member $\mathrm{H}$.
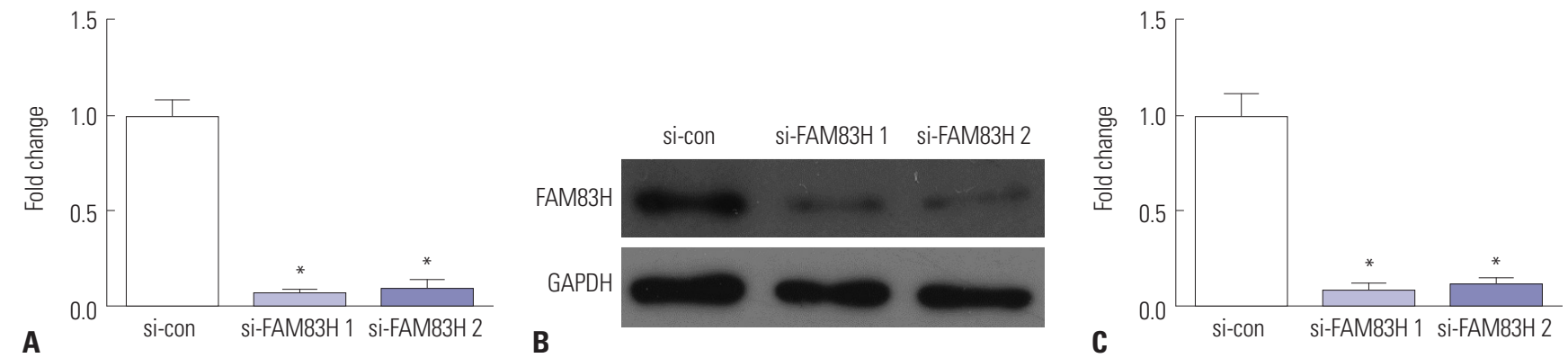

Fig. 2. FAM83H expression in HeLa cells transfected with siRNA FAM83H 1\# and siRNA FAM83H 2\# were measured by Real-Time Quantitative Reverse Transcription PCR $(\mathrm{A})$ and Western blotting $(\mathrm{B}$ and $\mathrm{C}) .{ }^{*} p<0.01$ compared to the si-con group. FAM $83 \mathrm{H}$, family with sequence similarity 83 member $\mathrm{H}$; GAPDH, glyceraldehyde-3-phosphate dehydrogenase. 


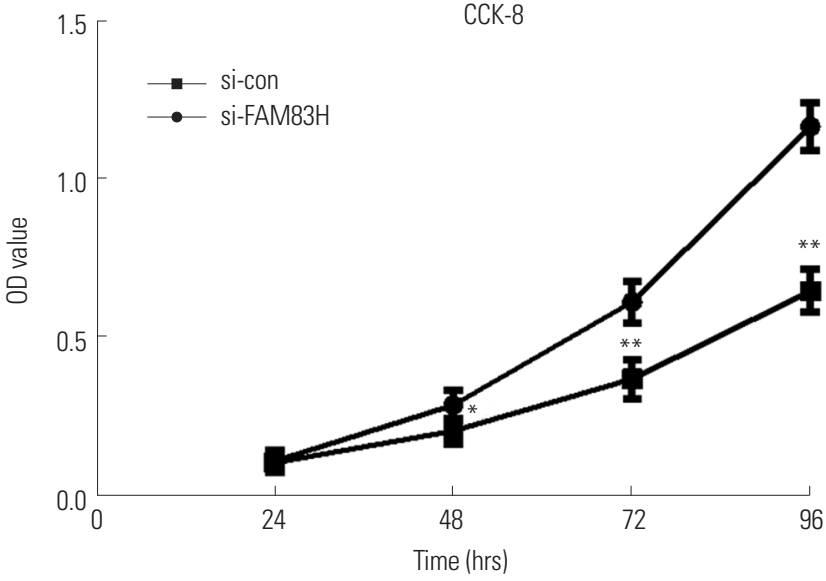

Fig. 3. Knockdown of FAM83H inhibits growth and viability of HeLa cells. CCK-8 assay showing viability of transfected HeLa cells at 24, 48, 72, and $96 \mathrm{~h}$. ${ }^{*} p<0.05$ compared to control, ${ }^{* *} p<0.001$ compared to control. CCK8, Cell-Counting Kit 8; FAM83H, family with sequence similarity 83 member $\mathrm{H}$; OD, optical density.

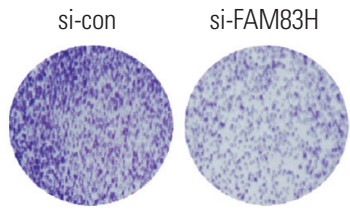

A

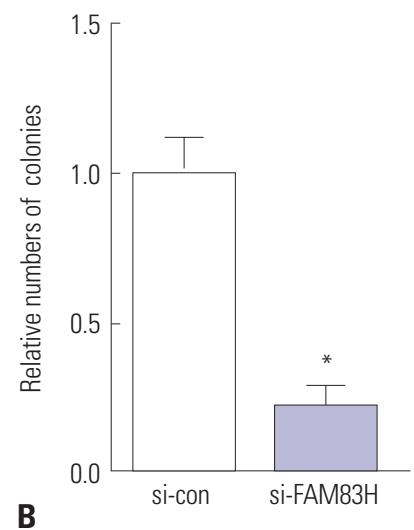

Fig. 4. Knockdown of FAM83H decreases colony formation of HeLa cells. (A) Representative images of colony formation assay $(\times 2)$. The clones were dyed by $0.1 \%$ crystal violet. (B) Quantification of the colony numbers. ${ }^{*} p<0.001$ compared to control. FAM $83 \mathrm{H}$, family with sequence similarity 83 member $\mathrm{H}$. the potential effects of FAM83H knockdown on cell migration and invasion. We observed that, after FAM83H silencing, the migration and invasion of CC cells were effectively inhibited $(p<0.001)$ (Fig. 5B and C). Overall, these data indicated that FAM83H promotes CC cell migration and invasion.

\section{Knockdown of FAM83H inhibits the activation of PI3K signaling pathway}

To further describe the specific molecular mechanism of FAM83H-regulated proliferation and migration of CC cells, we used Western blotting to explore the candidate targets of FAM83H, by which we selected the PI3K signaling pathway (AKT, p-AKT, PI3K, p-PI3K) as a target, since the PI3K pathway plays key roles in mediating proliferation and migration. ${ }^{14-16}$ Fig. 6 shows that knockdown of FAM83H remarkably inhibited the expression levels of p-AKT and p-PI3K in CC cells $(p<0.001)$, but did not influence total PI3K and AKT expression levels.

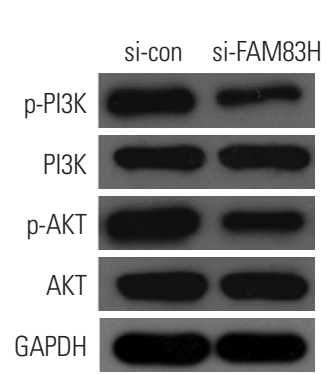

A

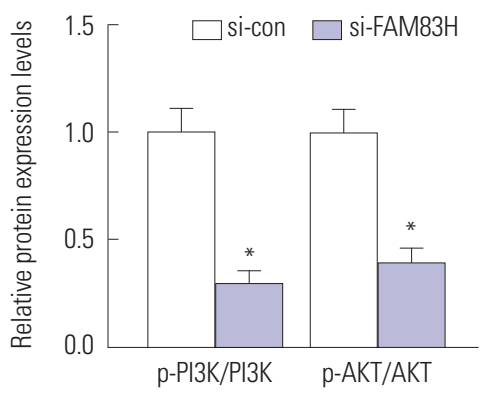

B
Fig. 6. The expression of PI3K/AKT pathway related proteins. After FAM83H silencing, the expression of $p-A K T$ and $p-P I 3 K$ were all reduced significantly. (A) The expression of PI3K pathway related proteins were determined by Western blot. (B) Quantification of the protein expression levels in (A). * $p<0.001$ compared to control. GAPDH, glyceraldehyde3-phosphate dehydrogenase; FAM83H, family with sequence similarity 83 member $\mathrm{H}$.

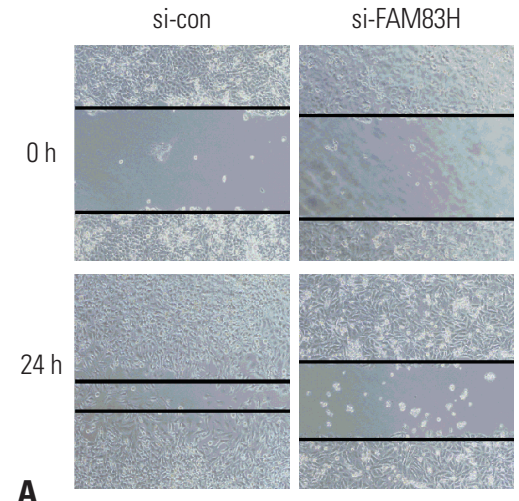

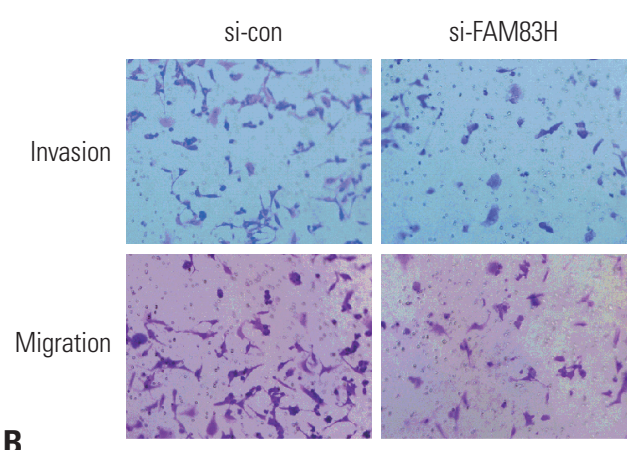

B

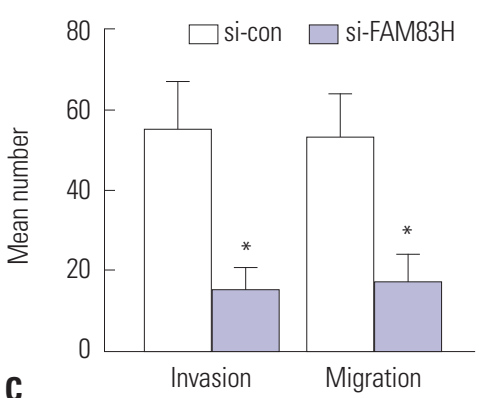

Fig. 5. Analysis of wound-healing $(A ; \times 100)$ and transwell assay $[B(\times 200)$ and $C]$. Cells were dyed by $0.1 \%$ crystal violet. In HeLa cells transfected with si-FAM83H, the invasion and migration were significantly decreased compared to the cells transfected with scrambled si-RNA (si-con). ${ }^{*} p<0.001$ compared to control. FAM83H, family with sequence similarity 83 member $\mathrm{H}$. 


\section{DISCUSSION}

In our study, we investigated the role of FAM83H in CC and provided evidence suggesting that FAM83H plays an oncogenic role in CC. The oncogenic role of FAM83H is supported by the expression of relatively higher levels of FAM83H in CC, compared with normal tissues obtained from TCGA database. Consistent with bioinformatics results, overexpression of FAM83H was also observed in CC cells. Additionally, high FAM83H expression was found to be closely correlated with shorter survival in CC patients. As FAM83H has been found to be overexpressed in multiple other tumor types (e.g., colorectal cancer), ${ }^{6,8}$ these data strongly support that FAM83H itself is an important oncogene in CC.

In depth, we studied the role and significance of FAM83H in CC cell proliferation and migration. Knockdown of FAM83H effectively inhibited the proliferation and growth of HeLa cells. Colony-formation assay demonstrated that the expression of FAM83H affects the colony-formation capacity of HeLa cells. Of note, a previous study demonstrated that knockdown of FAM83H in LNCaP cells suppressed colony formation. ${ }^{10}$ So far, FAM83H has been reported to mediate several target proteins in cell migration and proliferation. Meanwhile, studies have suggested that the PI3K pathway plays key roles in mediating proliferation and migration. ${ }^{14-16}$ Accordingly, we investigated whether PI3K was affected by FAM83H in CC cells using Western blotting.

PI3K/AKT signaling pathway has been implicated in the physiological functions of human malignant tumors, including ovarian, breast, and pancreatic cancer. ${ }^{17}$ AKT activity is triggered by PI3K, and AKT activation induces cell survival, migration, and metabolism through phosphorylation of various physiological factors. ${ }^{18}$ It is noteworthy that sustained AKT activity in CC cells (HeLa and Caski) may facilitate their growth and survival. ${ }^{19}$ Several studies have reported that the PI3K/AKT pathway is activated in CC. For example, in a Korean study of 27 CC patients (18 without and nine with recurrence), p-AKT expression was related to local recurrence after primary radiotherapy. ${ }^{20}$ Another study of 46 paraffin-embedded specimens without associated clinical outcome data, $\mathrm{p}$ AKT staining was positive in 39 (85\%) of the samples..$^{21} \mathrm{Liu}$, et al. ${ }^{22}$ suggested that inhibition of Akt/mTOR/p70S6K translational machinery results in the downregulation of MMP-9 and urokinase plasminogen activator, playing an anti-metastatic role. In the current study, Western blot analysis revealed significant decreases in the activity of p-AKT and p-PI3K in siFAM83H-transfected CC cells. Accordingly, we inferred that FAM83H promotes $\mathrm{CC}$ growth and migration, at least partially by mediating PI3K/AKT signaling.

To our best knowledge, this is the first study to report that FAM83H increases cell proliferation, colony formation, migration, and invasion of CC cells. Our work suggests that inhibition of FAM83H might be a potential therapeutic strategy in the management of CC. However, we must take into consideration several limitations. To begin with, we did not use clinical samples collected by ourselves to detect FAM83H expression in CC tissues. Secondly, animal models of tumors were not constructed to verify our results obtained in the cell experiments. Nevertheless, our study provides evidence of use to further exploring the molecular mechanisms of CC and developing candidate targets for the treatment thereof.

\section{AUTHOR CONTRIBUTIONS}

Conceptualization: Chao Chen and Jia Zhou. Data curation: Chao Chen and Hua-Feng Li. Formal analysis: Yu-Jie Hu. Investigation: Chao Chen, Hua-Feng Li, Yu-Jie Hu, Meng-Jie Jiang, and Qing-Sheng Liu. Methodology: Chao Chen. Project administration: Chao Chen and Jia Zhou. Software: Hua-Feng Li and Yu-Jie Hu. Supervision: Jia Zhou. Validation: Meng-Jie Jiang and Qing-Sheng Liu. Visualization: Chao Chen. Writing_original draft: Chao Chen. Writing_review and editing: Jia Zhou.

\section{ORCID iDs}

Chao Chen https://orcid.org/0000-0002-0342-4170

Hua-Feng Li https://orcid.org/0000-0001-9274-942X

Yu-Jie $\mathrm{Hu}$ https://orcid.org/0000-0001-6328-175X

Meng-Jie Jiang https://orcid.org/0000-0001-5129-676X

Qing-Sheng Liu https://orcid.org/0000-0003-1351-1443

Jia Zhou https://orcid.org/0000-0001-6143-9584

\section{REFERENCES}

1. Ili CG, Brebi P, López J, García P, Leal P, Suarez E, et al. Genotyping of human papillomavirus in cervical intraepithelial neoplasia in a high-risk population. J Med Virol 2011;83:833-7.

2. Chen W, Zheng R, Baade PD, Zhang S, Zeng H, Bray F, et al. Cancer statistics in China, 2015. CA Cancer J Clin 2016;66:115-32.

3. Lee SY, Meier R, Furuta S, Lenburg ME, Kenny PA, Xu R, et al. FAM83A confers EGFR-TKI resistance in breast cancer cells and in mice. J Clin Invest 2012;122:3211-20.

4. Wang Z, Liu Y, Zhang P, Zhang W, Wang W, Curr K, et al. FAM83D promotes cell proliferation and motility by downregulating tumor suppressor gene FBXW7. Oncotarget 2013;4:2476-86.

5. Cipriano R, Graham J, Miskimen KL, Bryson BL, Bruntz RC, Scott SA, et al. FAM83B mediates EGFR- and RAS-driven oncogenic transformation. J Clin Invest 2012;122:3197-210.

6. Snijders AM, Lee SY, Hang B, Hao W, Bissell MJ, Mao JH. FAM83 family oncogenes are broadly involved in human cancers: an integrative multi-omics approach. Mol Oncol 2017;11:167-79.

7. Kuga T, Sasaki M, Mikami T, Miake Y, Adachi J, Shimizu M, et al. FAM83H and casein kinase I regulate the organization of the keratin cytoskeleton and formation of desmosomes. Sci Rep 2016;6: 26557.

8. Kuga T, Kume H, Kawasaki N, Sato M, Adachi J, Shiromizu T, et al. A novel mechanism of keratin cytoskeleton organization through casein kinase I $\alpha$ and FAM83H in colorectal cancer. J Cell Sci 2013; 126(Pt 20):4721-31.

9. Kuga T, Kume H, Adachi J, Kawasaki N, Shimizu M, Hoshino I, et al. Casein kinase 1 is recruited to nuclear speckles by FAM83H and SON. Sci Rep 2016;6:34472.

10. Nalla AK, Williams TF, Collins CP, Rae DT, Trobridge GD. Lentivi- 
ral vector-mediated insertional mutagenesis screen identifies genes that influence androgen independent prostate cancer progression and predict clinical outcome. Mol Carcinog 2016;55: 1761-71.

11. Kim KM, Park SH, Bae JS, Noh SJ, Tao GZ, Kim JR, et al. FAM83H is involved in the progression of hepatocellular carcinoma and is regulated by MYC. Sci Rep 2017;7:3274.

12. Li L, Wang L, Song P, Geng X, Liang X, Zhou M, et al. Critical role of histone demethylase RBP2 in human gastric cancer angiogenesis. Mol Cancer 2014;13:81.

13. Jiang CG, Lv L, Liu FR, Wang ZN, Liu FN, Li YS, et al. Downregulation of connective tissue growth factor inhibits the growth and invasion of gastric cancer cells and attenuates peritoneal dissemination. Mol Cancer 2011;10:122.

14. Okano J, Shiota G, Matsumoto K, Yasui S, Kurimasa A, Hisatome I, et al. Hepatocyte growth factor exerts a proliferative effect on oval cells through the PI3K/AKT signaling pathway. Biochem Biophys Res Commun 2003;309:298-304.

15. Goncharova EA, Ammit AJ, Irani C, Carroll RG, Eszterhas AJ, Panettieri RA, et al. PI3K is required for proliferation and migration of human pulmonary vascular smooth muscle cells. Am J Physiol Lung Cell Mol Physiol 2002;283:L354-63.

16. Meng Q, Xia C, Fang J, Rojanasakul Y, Jiang BH. Role of PI3K and
AKT specific isoforms in ovarian cancer cell migration, invasion and proliferation through the p70S6K1 pathway. Cell Signal 2006;18:2262-71.

17. Blanco-Aparicio C, Renner O, Leal JF, Carnero A. PTEN, more than the AKT pathway. Carcinogenesis 2007;28:1379-86.

18. Kandel ES, Hay N. The regulation and activities of the multifunctional serine/threonine kinase Akt/PKB. Exp Cell Res 1999;253: 210-29.

19. Kim MJ, Lee TH, Kim SH, Choi YJ, Heo J, Kim YH. Triptolide inactivates Akt and induces caspase-dependent death in cervical cancer cells via the mitochondrial pathway. Int J Oncol 2010;37:117785.

20. Kim TJ, Lee JW, Song SY, Choi JJ, Choi CH, Kim BG, et al. Increased expression of pAKT is associated with radiation resistance in cervical cancer. Br J Cancer 2006;94:1678-82.

21. Bertelsen BI, Steine SJ, Sandvei R, Molven A, Laerum OD. Molecular analysis of the PI3K-AKT pathway in uterine cervical neoplasia: frequent PIK3CA amplification and AKT phosphorylation. Int J Cancer 2006;118:1877-83.

22. Liu SC, Chen C, Chung CH, Wang PC, Wu NL, Cheng JK, et al. Inhibitory effects of butein on cancer metastasis and bioenergetic modulation. J Agric Food Chem 2014;62:9109-17. 